\title{
Nonexponential relaxation of diluted antiferromagnets
}

\author{
U. Nowak and K. D. Usadel \\ Theoretische Tieftemperaturphysik, Universität Duisburg, Lotharstrasse 1, 4100 Duisburg 1, West Germany
}

(Received 15 June 1990)

\begin{abstract}
We report on extensive Monte Carlo simulations of diluted Ising-type antiferromagnets in a uniform magnetic field in three dimensions. The analysis of the relaxation data of the domain state leads to a power-law decay of the remanent magnetization and internal energy. The corresponding exponents are proportional to the temperature. The power noise spectrum is investigated, showing roughly $1 / f$ noise.
\end{abstract}

In recent years, there have been several theoretical and experimental studies on the behavior of random-field systems. $^{1}$ Experiments are often performed on diluted Ising-type antiferromagnets in a uniform external magnetic field (DAFF) which belong to the same universality class as the random-field Ising model (RFIM). ${ }^{2}$

Like the RFIM, the DAFF develops a metastable domain state if the system is cooled in a field from the paramagnetic phase. ${ }^{3}$ The domain state relaxes towards long-range order after switching off the field. This relaxation is very slow because of pinning of the domain walls at vacancies. Since the domain state carries a surplus magnetization, a slow decay of the remanent magnetization can be observed. Experimentally the remanent magnetization has been studied in detail by Kleemann et al. ${ }^{4}$ They found a $[\ln (1 / \tau)]^{-\Gamma}$ law for the decay of the magnetization. Since no growth of the domains was found even after switching off the field, ${ }^{1}$ Nattermann and Vilfan $^{5}$ explained this very slow relaxation assuming a domain-wall reorientation.

In an earlier publication we investigated the mechanisms for the relaxation of the diluted antiferromagnet by a Monte Carlo simulation. ${ }^{6}$ The simulation showed that the relaxation primarily takes place in the domain walls for low temperatures, confirming the ideas put forward by Nattermann and Vilfan. ${ }^{5}$ For higher temperature, however, a significant growth of the domains was found.

From the results of our simulations we could not distinguish whether the decay of the magnetization follows a logarithmic law or, for instance, a power law. With both of these laws the data could be fitted with practically the same degree of accuracy. Since the physics behind these laws seem to be quite different, it is very desirable to distinguish between them. Therefore, in the meantime we increased the time of a Monte Carlo run by factors of up to 40 compared to our earlier investigation; i.e., we made Monte Carlo runs with up to 100000 Monte Carlo steps per spin (MCS's). Even with this long computation time a very clear distinction between power-law decay and the logarithmic law was difficult to achieve, although an extensive configurational averaging-performed only for one simulation because of the very long computation times-confirmed the power law to be correct.

An interesting new observation is a structure in the fluctuations of the unaveraged magnetization data. It appears that these fluctuations are present on all time scales, of course within a certain time window, which is dictated by the length of the Monte Carlo run. This interesting observation leads to a new kind of analysis of the magnetization data which also will be reported here.

As in our earlier work we performed Monte Carlo simulations on a three-dimensional simple cubic lattice with a size of $61 \times 61 \times 60$ and a dilution of $50 \%$. The Hamiltonian in units of the coupling constant $J$ reads

$$
H=\sum_{\langle i, j\rangle} \varepsilon_{i} \varepsilon_{j} \sigma_{i} \sigma_{j}-B \sum_{i} \varepsilon_{i} \sigma_{i},
$$

where $\sigma_{i}= \pm 1$ and $\varepsilon_{i}=0.1$. Only nearest-neighbor interaction is considered. We used helical boundary conditions and the heat-bath algorithm. ${ }^{7}$ The domain state was prepared by performing 1000 MCS's at a particular temperature $T$ and a field $B$ starting from a completely random spin configuration. Within this time the random spin configuration relaxes to a practically frozen domain state which carries a surplus magnetization. This state serves as the initial configuration for the computation of the remanent magnetization and the internal energy.

Analyzing our data we observed that fluctuations of the remanent magnetization which made the data fitting ambiguous in the earlier simulations occur in the longer simulations as well, but now also on longer scales. Therefore we extended the investigation in two directions. On one hand we carried out a configurational averaging in order to decrease the fluctuations as far as necessary in order to distinguish between power law and logarithmic law. On the other hand, we investigated the temperature and field dependence and the fluctuations of the unaveraged remanent quantities.

Figure 1 shows a log-log plot of the remanent magnetization for a temperature $T=0.4$ and an initial field $B_{i}=2.0$ averaged over six realizations of the lattice. It is obvious that the decay is described very well by a power law $M(t) \sim t^{-\beta}$. In comparing the mean-square deviations for fitting the data by a power law or a logarithmic law, respectively, the deviation of the power law turns out to be ten times smaller. This extensive averaging was performed only once due to the very long computation time needed (the simulation of Fig. 1 took about $60 \mathrm{~h}$ on 


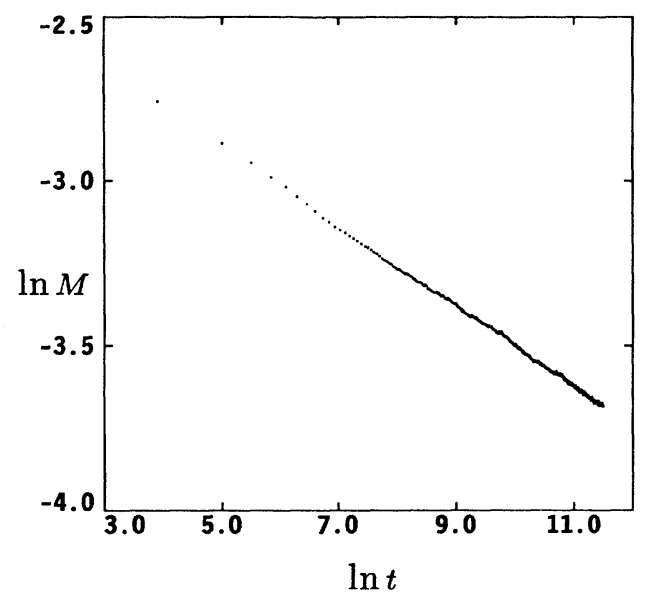

FIG. 1. Decay of the remanent magnetization $\ln M$ vs $\ln t$ averaged over size systems.

a convex $\mathrm{C} 220$ vector computer).

Figure 2 shows a log-log plot of the unaveraged magnetization versus time. The upper curve shows the magnetization of the first 1000 MCS's after switching off an external field of $B_{i}=2.0$ at a temperature $T=0.8$. The intermediate curve shows the magnetization of the first 10000 MCS's of the same run rescaled in time by a factor of 10 , i.e., every point is an average of ten consecutive MCS's. The lower curve is again rescaled by a factor of 10 and every point is an average of 100 consecutive MCS's. The results obtained in Fig. 2 are typical in the sense that similar behavior is obtained for different temperatures and magnetic fields.

From these results we are led to conclude that there exists no typical time scale in the relaxation process. There are relaxation processes on all time scales leading to a fluctuating magnetization $M(t)$ which has a self-similar

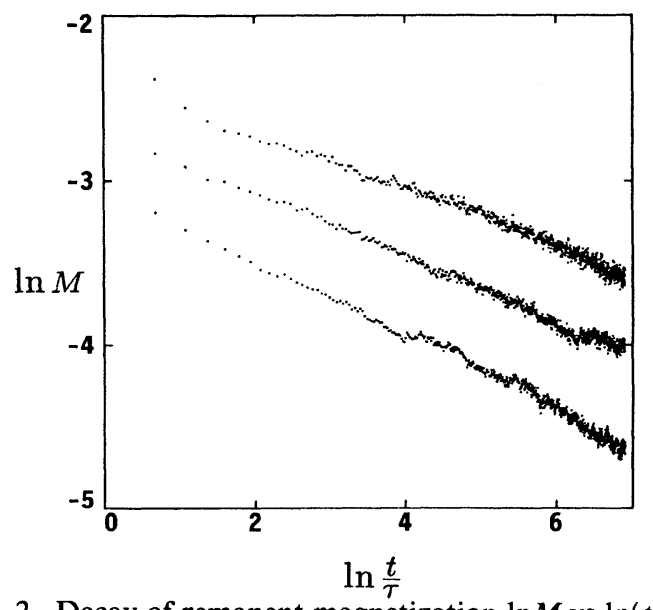

FIG. 2. Decay of remanent magnetization $\ln M$ vs $\ln (t / \tau)$ for different time scales $\tau=1$ (upper curve), 10 (middle curve), 100 (lower curve).

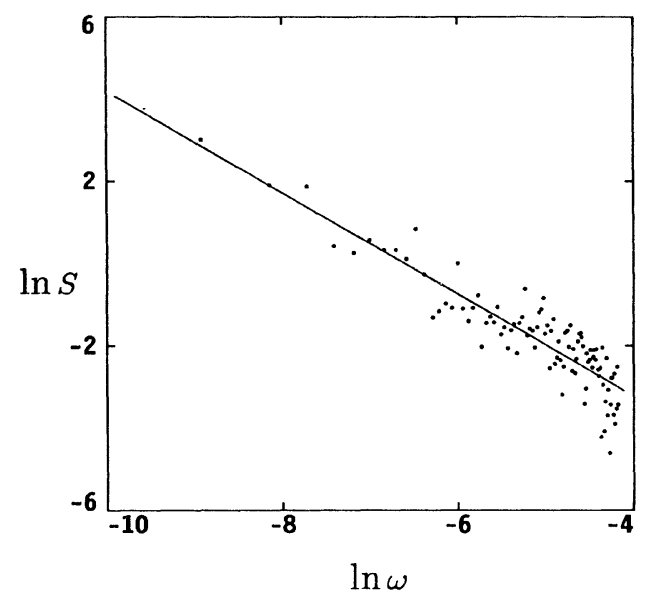

FIG. 3. Square of the Fourier transform of the noise data $S$ vs $\omega$ for $T=0.6, H_{i}=2.0$. The straight line is best fitted.

structure, i.e., it shows on all time scales $\tau$ the same qualitative behavior. The lack of a typical time scale in our opinion is due to a lack of a typical length scale in the system. Above the percolation threshold $p_{c}$ for the vacancies (31\% vacancies, i.e., $69 \%$ magnetic ions) there exists a percolating vacancy cluster which is a fractal below a length scale $\xi \sim\left(p-p_{c}\right)^{-v} .{ }^{8}$ Apart from this, the domain wall by itself is a fractal, as has been shown by Cambier and Nauenberg ${ }^{9}$ for the RFIM. The relaxation of the domain state takes place in the domain wall or-in the case of domain growth-is caused by movements of the domain wall. ${ }^{6}$ Due to the fractality of the domain wall as well as the fractality of the vacancy clusters there is a lack of typical length scales in the system which leads to a wide range of energy barriers with long-range temporal correlations resulting in a lack of typical time scales in the relaxation. Trivially, a law without a typical time

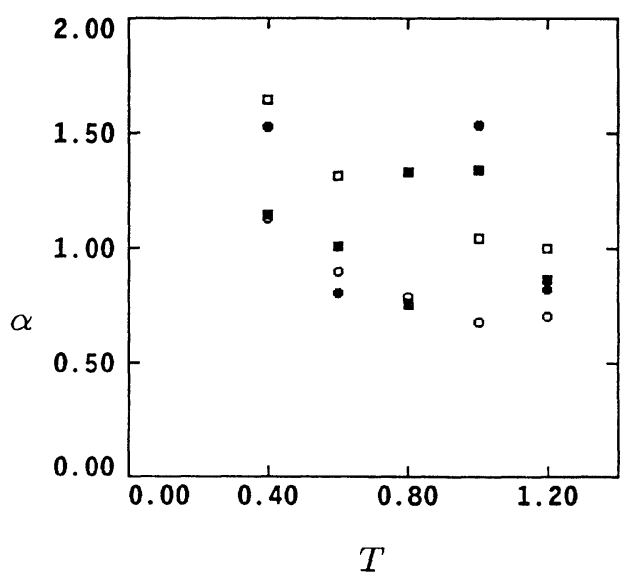

FIG. 4. Exponent $\alpha$ vs temperature for remanent magnetization (solid symbols) and internal energy (open symbols) for initial fields of 2.0 (squares) and 1.5 (circles). 
scale is a power law.

It is therefore very interesting to analyze the noise spectrum of our simulation data. Therefore we have calculated the power spectrum of the noise data $S(\omega)$ :

$$
S(\omega)=\left|\int e^{i \omega t} \Delta M(t) d t\right|^{2},
$$

where $\Delta M(t)$ is the deviation of magnetization data from the best-fitted power law. A similar analysis was performed for the internal energy $\Delta E$. Figure 3 shows the $\log -\log$ plot of $S(\omega)$ for a temperature of 0.6 and a field $B_{i}$ of 2.0. The possible values for $\omega$ varied from $\omega_{\min }=2 \pi / T$ to $\omega_{\max }=\pi / \Delta t$, where $T$ is the simulation time (100000 MCS's for the long time simulation) and $\Delta t$ is the smallest computed time step (100 MCS's). Even the shorter simulations have been analyzed ( $T=1000$ MCS's, $\Delta t=1 \mathrm{MCS})$ with essentially the same results. In Fig. 3 we have averaged the data of $S(\omega)$ over slightly different $\omega$. Unfortunately the data are quite unexact, and therefore the exponent $\alpha$ with $S(\omega) \sim \omega^{-\alpha}$ extracted from them is only roughly determined. Figure 4 shows this exponent $\alpha$ obtained from the remanent magnetization (solid symbols) and internal energy (open symbols) for five different temperatures and two different fields. The values of $\alpha$ fluctuate around $\alpha \approx 1$. No systematical temperature dependence of $\alpha$ is observed, and both the magnetization and the internal energy give practically the same exponent $\alpha$.

Figure 5 shows the exponent $\beta$ of the power law for the decaying magnetization and internal energy for the same temperatures and fields as in Fig. 4. $\beta$ is roughly proportional to the temperature-a relation also observed in spin glasses ${ }^{10}$-and depends only slightly on the field. Apart from this proportionality, the calculated values of $\beta$ for the decay of the magnetization and the internal energy are different.

Here some considerations about the differences between the experimental ${ }^{4}$ and theoretical ${ }^{5}$ findings and our simulations are in order. High magnetic fields $B \sim J$ involve small domains. Of course we have to use high fields in the simulation since the domains have to be smaller than the simulated lattice. The experimentally investigated regime is that of low fields $(B<<J)$ where the domains have a radius of several hundred lattice constants much more than the scale below which the domains are fractal, although the surface may be fractal on small scales. This

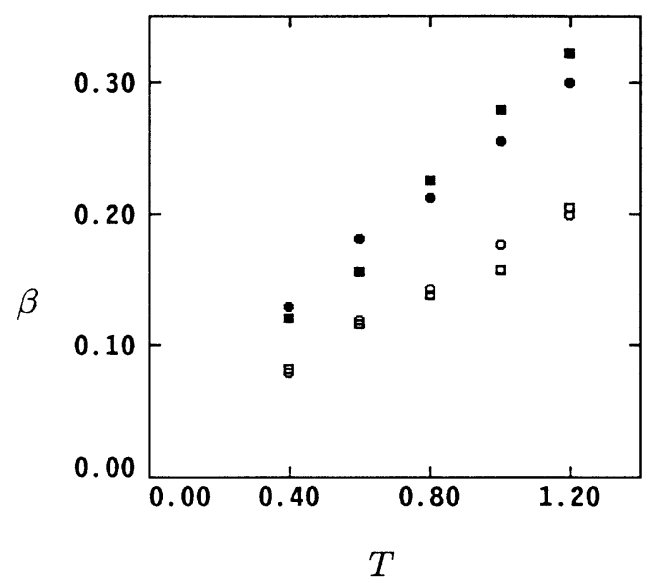

FIG. 5. Exponent $\beta$ vs temperature as explained in Fig. 4.

is the regime described by Nattermann and Vilfan in the low-temperature limit, i.e., under the assumption that no domain growth occurs. ${ }^{6}$ In our simulation we investigated the regime of small length scales where the domains are fractal and where a distinction between domain-wall movements and domain growth is hardly possible.

Very recently we were informed about new experiments at higher magnetic fields ${ }^{11}$ confirming our result that the power law describes the decay of the remanent magnetization for higher initial fields.

Finally we would like to emphasize that in our opinion there could be a connection between the relaxation of the domain state of diluted antiferromagnets and the theory of self-organized criticality. ${ }^{12}$ This theory explains the occurrence of fractality in nature as the critical point of the dynamics of dissipative systems with long-range temporal and spatial correlation and $1 / f$ noise. Most of the considerations of Bak et al. can be transferred to our simulations so that it is tempting to consider the relaxing antiferromagnet to be in a state of self-organized criticality, ${ }^{13}$ although further simulations are needed to clarify the situation.

We would like to thank D. P. Belanger and W. Kleemann for informing us of their results prior to publication. This work was supported by the Deutsche Forschungsgemeinschaft through Sonderforschungsbereich 166 .
${ }^{1}$ For reviews, see R. J. Birgeneau, R. A. Cowley, G. Shirane, and H. Yoshizawa, J. Stat. Phys. 34, 817 (1984); D. P. Belanger, S. M. Rezende, A. R. King, and V. Jaccarino, J. Appl. Phys. 57, 3294 (1985); R. A. Cowley, R. J. Birgeneau, and G. Shirane, Physica 140A, 285 (1986).

${ }^{2}$ S. Fishman and A. Aharony, J. Phys. C 12, L729 (1979).

${ }^{3}$ G. S. Grest, C. M. Soukoulis, and K. Levin, Phys. Rev. B 33, 7659 (1986)

${ }^{4}$ U. A. Leitão, W. Kleemann, and I. B. Ferreira, Phys. Rev. B 38, 4765 (1988); P. Pollak, W. Kleemann, and D. P. Belanger, ibid. 38, 4773 (1988).

${ }^{5}$ T. Nattermann and I. Vilfan, Phys. Rev. Lett. 61, 223 (1988).

${ }^{6}$ U. Nowak and K. D. Usadel, Phys. Rev. B 39, 2516 (1989).
${ }^{7}$ K. Binder and D. W. Heermann, Monte Carlo Simulations in Statistical Physics (Springer-Verlag, Berlin, 1988).

${ }^{8}$ C. Domb and J. L. Lebowitz, Phase Transitions and Critical Phenomena (Academic, London, 1988).

${ }^{9}$ J. A. Cambier and M. Nauenberg, Phys. Rev. B 34, 7998 (1986).

${ }^{10}$ J. Ferré, J. Rajchenbach, and H. Maletta, J. Appl. Phys. 52, 1697 (1981).

${ }^{11}$ W. Kleemann and D. P. Belanger (private communications).

12P. Bak, C. Tang, and K. Wiesenfeld, Phys. Rev. Lett. 59, 381 (1987).

${ }^{13}$ U. Nowak and K. D. Usadel, Physica B 165, 211 (1990). 